\title{
Arbeitszeitgesetz nach EuGH - Was nun?
}

A m 9. September 2003 erlangte ein Kieler Kollege vor dem Europäischen Gerichtshof (EuGH in Luxemburg) das Urteil, dass Bereitschaftsdienste im Krankenhaus in vollem Umfange Arbeitszeit seien. Dieses Urteil löste Erleichterung und Freude, aber auch Skepsis aus: Erleichterung, weil den kräftezehrenden übermäßigen Arbeitsbelastungen der Klinikärzte ein Ende gesetzt würde, Skepsis, weil sich wohl vorerst kaum etwas ändern wird. Zunächst gilt das Urteil auch nur für Ärzte mit öffentlich rechtlichen Arbeitsverträgen, denn europäische Richtlinien greifen bei Verträgen mit privaten Arbeitgebern nicht unmittelbar.

Keiner weiß genau, wie die Entscheidung des Europäischen Gerichtshofs in den deutschen Kliniken wirklich genau umgesetzt werden könnte. Betroffen sind mehr als 140000 Klinikärzte. Wie Berechnungen ergeben, wären etwa 15000 Neueinstellungen nötig. Sollte ein Zwei- oder besser auch ein Drei-Schicht-Modell eingeführt werden, wäre nach Auskunft der Deutschen Krankenhausgesellschaft (DKG) sogar die Einstellung von 27000 Ärzten erforderlich. Dies wiederum würde Kosten von knapp zwei Milliarden Euro nach sich ziehen. Und ein weiteres Problem: So viele Ärzte gibt der Arbeitsmarkt zurzeit nicht her.

Jedoch steht mit diesem Urteil fest, dass die momentan üblichen langen Dienstzeiten von bis zu 30 Stunden ab sofort illegal sind. Künftig darf die Arbeitszeit für Klinikärzte maximal 48 Stunden pro Woche betragen. Sofern Arbeitszeiten von mehr als zwölf Stunden anfallen, ist eine mindestens elfstündige Ruhezeit einzuhalten. Die tägliche Arbeitszeit sollte acht Stunden nicht überschreiten, und ein Ausgleich für Mehrarbeit sollte innerhalb von zwölf Monaten erfolgen. Zusätzlich sind aber außerhalb dieser Regelung Ausnahmeverträge auch ohne Zeitausgleich möglich.

Interessanterweise wurde vor kurzem der Chefarzt der Chirurgie des Krankenhauses Burgwedel wegen eines Verstoßes gegen das Arbeitszeitgesetz von seiner Bezirksregierung zu 35 Euro Strafe verurteilt. Die Anzeige eines Assistenten hatte zu Ermittlungen des Gewerbeaufsichtsamtes geführt, das 22 Fälle von Arbeitsüberschreitungen mit einer halben bis zu einer Stunde (!) feststellte. Aufgrund dieser Delikte forderte die Bezirksregierung von dem Mediziner - der ja laut Krankenhausverwaltung für die Einteilung der Arbeitszeiten zuständig ist - das oben erwähnte Bußgeld. Der
Verwaltungsdirektor des gleichen Krankenhauses sollte ein Bußgeld von 1700 Euro an die Behörde bezahlen. Er stritt seine Schuld jedoch ab und ging vor Gericht. Das Amtsgericht Hannover hat das Verfahren gegen den Verwaltungsdirektor eingestellt. Die Diskrepanz zwischen den beiden geforderten Beiträgen hatte Gericht und Staatsanwalt nicht eingeleuchtet. Der Chirurg hat - so wurde berichtet - sein Bußgeld bezahlt.

Es wäre schlimm, wenn durch die Folgen des Urteils des Europäischen Gerichtshofes Unfrieden zwischen der Ärzteschaft und den Verwaltungen im Krankenhaus zu einem Dauerthema würde. Letztendlich muss die Problematik von der Politik gelöst werden. Die vorgelegte Nachbesserung des geplanten Arbeitsmarktreformgesetzes wird hierzu jedoch sicherlich bei weitem nicht ausreichen.

Um einen andauernden Konflikt zu vermeiden, sollten wir einen positiven innerbetrieblichen Dialog in Gang setzen. Nur so können wir die Illegalität (bezogen auf das Arbeitszeitgesetz) verhindern und trotzdem zumutbare Arbeitsverhältnisse und eine sichere, gewohnt gute Patientenversorgung gewährleisten. Hierzu sind offene Gespräche erforderlich, bei denen sich keine Seite auf das hohe Ross setzen darf. Denn Fakt ist auch, dass ganz speziell nach der Einführung der so genannten „diagnosis related groups“ (DRGs) viele Krankenhausbetriebe sonst rasch nicht mehr konkurrenzfähig sein werden.

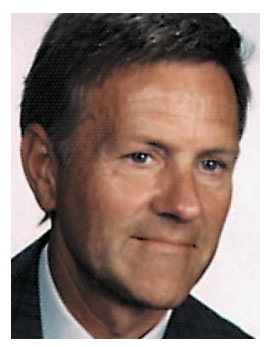

Prof. Dr. B. Stegemann, Hagen

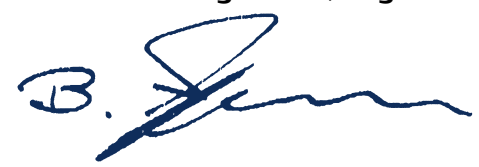

